\title{
Hyperpulsatile pressure, systemic inflammation and cardiac stress are associated with cardiac wall remodeling in an African male cohort: the SABPA study
}

\begin{abstract}
Esmé Jansen van Vuren ${ }^{1}$, Leoné Malan ${ }^{1}$, Roland von Känel ${ }^{1,2}$, Marike Cockeran ${ }^{3}$ and Nicolaas T Malan ${ }^{1}$
Inflammation may contribute to an increase in cardiac wall stress through pathways related to cardiac remodeling. Cardiac remodeling is characterized by myocyte hypertrophy, myocyte death and modifications of the extracellular matrix. We sought to explore associations among cardiac remodeling, inflammation and myocardial cell injury in a bi-ethnic cohort of South African men and women. We included 165 men (76 African and 89 Caucasian) and 174 women (80 African and 94 Caucasian) between 20 and 65 years of age. Inflammatory markers used were C-reactive protein (CRP), interleukin-6 and tumor necrosis factor-alpha (TNF- $\alpha$ ), whereas troponin T (Trop T) and the N-terminal of pro B-type natriuretic peptide (NT-proBNP) were used as cardiac markers. The frequency of ischemic events (ST segment depression) and left ventricular strain (left ventricular hypertrophy: LVH) were monitored by a 24-h recording of ambulatory blood pressure (BP), ECG and 12-lead standard ECG. Hypertension diagnosed with ambulatory monitoring was more frequent in Africans (53.85 vs. 24.59\%; $P<0.001)$, as was the number of ischemic events $(6 \pm 15(1 ; 5)$ vs. $3 \pm 6(0 ; 3))$. Inflammatory markers (CRP, IL- 6 and TNF- $\alpha$ ) and the degree of LVH were all significantly higher in Africans $(P<0.05)$. BP was associated $(P<0.05)$ with Trop $\mathrm{T}$ in men across ethnic groups. In African men, cardiac stress (NT-proBNP) was associated with TNF-alpha $(P<0.001)$, Trop $\mathrm{T}(P<0.001)$ and pulse pressure $\left(P=0.048\right.$; adjusted $\left.R^{2}=0.45\right)$. The susceptibility for cardiac wall remodeling appears to increase with hyperpulsatile pressure, low-grade systemic inflammation and ventricular stress, and may lead to the development of future cardiovascular events in African men.

Hypertension Research (2016) 39, 648-653; doi:10.1038/hr.2016.45; published online 12 May 2016
\end{abstract}

Keywords: Africans; arterial tone; cardiac remodeling; cardiac troponin; inflammation

\section{INTRODUCTION}

Low-grade inflammation has been shown to be involved in the pathogenesis and progression of structural wall remodeling in Africans. ${ }^{1}$ Cytokines have a role in the inflammatory response by regulating hematopoiesis as well as immune and vascular reactions. ${ }^{2,3}$ A greater degree of endothelial dysfunction related to inflammation has been reported in African-Americans. ${ }^{4}$ One key pro-inflammatory cytokine in this process is tumor necrosis factor-alpha (TNF- $\alpha){ }^{5}$ The binding of TNF- $\alpha$ to its TNFR1 receptor may induce apoptosis in many cell types, including cardiac myocytes. ${ }^{6,7}$

Troponin $\mathrm{T}$ (Trop T) is a subunit of the troponin complex released in response to myocyte necrosis. ${ }^{8-10}$ Trop T levels have been found to be increased in African-American men. However, this association may be explained by the independent associations found between Trop
$\mathrm{T}$ and left ventricular hypertrophy (LVH). ${ }^{11}$ It has been shown that left ventricular structural changes or cardiac remodeling are associated with both inflammation and silent ischemia in African populations. ${ }^{1,12,13}$ A decrease in the metabolic supply to the myocardial tissue may result in ischemia of the myocardium. ${ }^{14}$ Persistent ischemia may result in irreversible cell death and migration of the inflammatory cytokines to the ischemic myocardium.

Studies have also shown that inflammation leads to an increase in arterial stiffness, the main cause of an increased pulse pressure (PP) or hyperpulsatile pressure. ${ }^{15,16}$ This increase in the hemodynamic load, which is accompanied by structural changes in the left ventricle, may lead to an increase in cardiac wall stress. ${ }^{8,17,18}$ The $\mathrm{N}$-terminal portion of pro-brain natriuretic peptide (NT-proBNP) is released in response to an increase in cardiac wall stress following myocyte stretching,

${ }^{1}$ Hypertension in Africa Research Team (HART), School for Physiology, Nutrition, and Consumer Science, North-West University, Potchefstroom, South Africa; ${ }^{2}$ Department of Neurology, IInselspital, Bern University Hospital, University of Bern, Bern, Switzerland and ${ }^{3}$ Medicine Usage in South Africa (MUSA), North-West University, Potchefstroom, South Africa

Correspondence: Professor L Malan, Hypertension in Africa Research Team (HART), School for Physiology, Nutrition, and Consumer Science, North-West University, Potchefstroom Campus, Private Bag X6001, Potchefstroom 2520, South Africa.

E-mail: Leone.Malan@nwu.ac.za

Received 26 November 2015; revised 8 March 2016; accepted 11 March 2016; published online 12 May 2016 
hypoxia and neuro-hormonal activation. ${ }^{18,19}$ NT-proBNP has been shown to be increased in African men, although the extent to which cultural and socioeconomic status may account for this ethnic difference is unclear. ${ }^{20,21}$ Significant positive associations between NT-proBNP and systolic blood pressure (SBP), PP and C-reactive protein (CRP) have also been demonstrated in African men, but the relation between NT-proBNP and other inflammatory markers (IL-6 and TNF- $\alpha$ ) still needs to be elucidated in African populations. ${ }^{21}$

Hence, we sought to determine whether associations exist among hyperpulsatile pressure, inflammation (assessed by CRP, IL-6, TNF- $\alpha$ levels), cardiac stress (assessed by NT-proBNP levels) and cardiac remodeling (assessed by LVH) in a bi-ethnic South African male and female cohort to accommodate cultural and behavioral differences. We specifically hypothesized that there would be positive associations among hyperpulsatile pressure, inflammation, cardiac stress and cardiac remodeling (LVH) in the African cohort.

\section{METHODS}

\section{Study design and participant selection}

In 2008 and 2009 (February to May), 2170 urban Caucasian and African teachers (aged 20-65 years) were invited to participate in the Sympathetic activity and Ambulatory Blood Pressure in Africans (SABPA) study. ${ }^{22}$ Teachers resided in the Dr Kenneth Kaunda Education District of the North West Province of South Africa. We ultimately enrolled 409 participants in the study (Figure 1). This selection was made to ensure that the participants were from a similar socioeconomic class. ${ }^{22}$

The exclusion criteria for the SABPA study were pregnancy, lactation, use of $\alpha$ - and $\beta$-blockers or psychotropic substances, blood donation or vaccinations 3 months prior to clinical assessment and a tympanum temperature exceeding $37.5^{\circ} \mathrm{C}$. Additional exclusions were made to avoid bias pertaining to cardiometabolic and inflammatory risk, and participants with an HIV-positive status $(N=19)$, clinically diagnosed diabetes mellitus $(N=10)$, anti-inflammatory medication usage $(N=24)$, anti-coagulant medication usage $(N=2)$, aspirin usage $(N=11)$ and history of myocardial infarction or stroke $(N=4)$ were excluded. ${ }^{23}$ After these exclusions, 165 men (76 African and 89 Caucasian) and 174 women (80 African and 94 Caucasian) participants remained in the study.

Informed consent was obtained from all the participants prior to the commencement of the study. The Ethics Review Board of North-West University, Potchefstroom Campus (NWU-00036-07-S6) gave ethical approval, and the study also complied with the Declaration of Helsinki's ethical guidelines (revised in 2008). ${ }^{24}$

\section{Experimental methods and data collection}

Research procedure. Clinical assessments were obtained over $36 \mathrm{~h}$. Cardiotens CE120 (Meditech, Budapest, Hungary) and accelerometers were used to

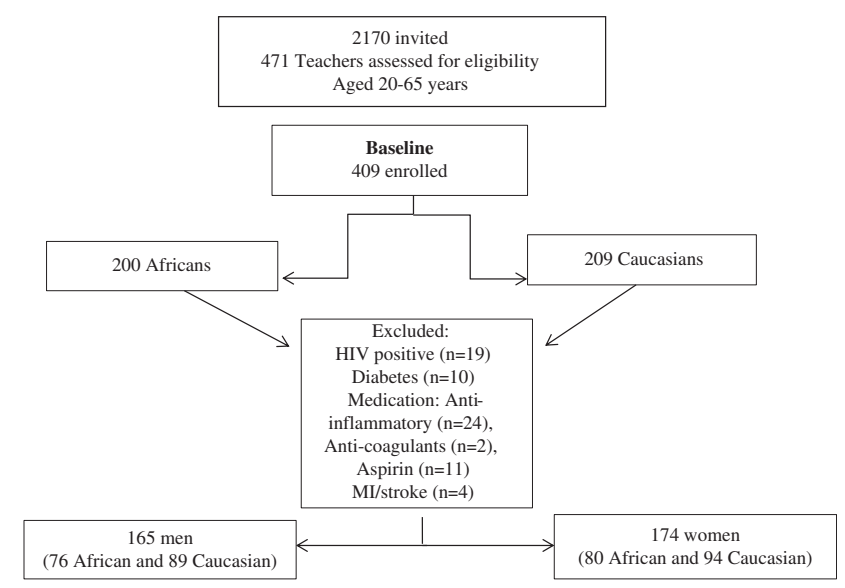

Figure 1 A South African bi-ethnic cohort. measure 24-h ambulatory BP, 2-lead ECG as well as 24-h physical activity every workday between $\sim$ 07:00 and 08:00. The correct cuff sizes were placed on the non-dominant arm of each participant; thereafter, the participants carried on with their normal daily activities.

At approximately 16:30, the participants were transported to the Metabolic Unit Research Facility at North-West University. The facility was well ventilated and maintained at a comfortable temperature, and each participant received his/her own private bedroom. The following day's procedures and experimental setup were explained to all the participants. Demographic and General Health questionnaires were completed, and each participant received a standardized dinner. The participants were advised to fast and rest from 22:00 for the next day's clinical measurements.

At approximately 06:00 in the next morning, the 24-h ambulatory apparatuses were disconnected, and the anthropometric and clinical measurements commenced. All resting ECGs and blood sampling were performed after the participants had been in a semi-recumbent position for approximately $30 \mathrm{~min}$. Upon the completion of all the assessments, the participants received breakfast and feedback and were then transported back to their respective schools.

Lifestyle determinants. The participants' daily physical activity was monitored over 24-h with an Actical activity monitor (Mini Mitter Co., Inc., Bend, OR, USA; Montreal, Quebec, QC, Canada). Anthropometric measurements were taken according to standardized procedures by registered level II anthropometrists in triplicate, and the mean of the three measurements was used to ensure accuracy. Inter- and intra-observer variability was found to be less than $10 \%$. The Mosteller formula of (weight $(\mathrm{kg}) \times$ height $(\mathrm{cm}) \div 3600) .1 / 2$ was used to calculate the body surface area..$^{25}$

Biochemical measurements. Registered nurses obtained fasting blood samples from the ante-brachial vein with a sterile winged infusion set. These samples were separated and stored at $-80^{\circ} \mathrm{C}$ until analysis. Gamma-glutamyl transferase $(\gamma$-GT), an indicator of alcohol abuse, was analyzed with the enzyme rate method (Unicel DXC 800; Beckman and Coulter, Krefeld, Germany). Serum cotinine, an indicator of nicotine levels, was analyzed with a homogeneous immunoassay that was performed on an Automated Modular system (Roche, Basel, Switzerland). ${ }^{26,27}$

Ultra-high-sensitivity CRP was measured with the turbidimetric method (Unicel DXC 800, Beckman and Coulter). Serum IIL-6 and high sensitive TNF- $\alpha$ were analyzed with the Human Il-6 Quantikine high sensitivity Enzyme-linked immunosorbent assay (HS ELISA; R\&D Systems, Minneapolis, MN, USA) and the Quantikine High Sensitivity Human Tumor TNF- $\alpha$ enzyme-linked immunosorbent assay (HS ELISA; R\&D Systems), respectively. The inter- and intra-assay variability for TNF- $\alpha$ were $15 \%$ and $17.8 \%$, respectively. For IL-6, the inter- and intra-assay variability were $18.9 \%$ and $5.9 \%$, respectively.

High-sensitive Trop T and NT-proBNP were analyzed with an electrochemiluminescence immunoassay (Elecsys, 2010, Roche). In our sample, there were $91(26.84 \%)$ undetectable Trop $\mathrm{T}$ values $\left(<3 \mathrm{pg} \mathrm{ml}^{-1}\right)$, which were substituted using the method of Croghan and Egeghy ${ }^{28}$ for values below the detection limit. The inter- and intra-batch variability was $15 \%$ and $5.6 \%$ for Trop T, and $4.6 \%$ and $4.2 \%$ for NT-proBNP, respectively.

Cardiovascular assessment procedures. The 24-h BP was measured at 30-min intervals from 08:00 to 22:00 and at 60-min intervals from 22:00 to 06:00. The successful inflation rates were $75.7 \%$ in African men, $84.9 \%$ in Caucasian men, $69.5 \%$ in African women and $85.8 \%$ in Caucasian women. The European Society of Hypertension (ESH) criteria for hypertension were used (average $24-\mathrm{h} \mathrm{SBP}$ of $\geqslant 130 \mathrm{~mm} \mathrm{Hg}$ and diastolic blood pressure (DBP) of $\geqslant 80 \mathrm{~mm} \mathrm{Hg}$ ). ${ }^{22}$ Silent ischemia (ambulatory ischemic events profile) was assessed with two-channel ECG recordings (pre-set program recording for $20 \mathrm{~s}$ at 5-min intervals). An ischemic event was recorded according to the 1-1-1 rule/criteria: horizontal or descending ST segment; depression by $1 \mathrm{~mm}$; duration of the ST segment episode of $1 \mathrm{~min}$, and there was a 1-min interval from the preceding episodes. ${ }^{13,29}$ The data were analyzed with the CardioVisions 1.19 Personal Edition software (Meditech). Throughout the day, the participants recorded any abnormalities that they experienced on a 24-h diary card. The abnormalities included visual disturbances, headaches, nausea, fainting, palpitations and 
Table 1 Comparing characteristics (mean \pm s.d.) between Africans and Caucasians

\begin{tabular}{|c|c|c|c|c|c|}
\hline Variables & Africans $(\mathrm{N}=156)$ & Median (IQ range) & Caucasians $(\mathrm{N}=183)$ & Median (IQ range) & P-values \\
\hline \multicolumn{6}{|l|}{ Confounders } \\
\hline Age, years & $44.33 \pm 8.28$ & $44.00(13.00)$ & $44.46 \pm 11.10$ & $47.00(14.00)$ & 0.907 \\
\hline Physical activity, kcal per day & $2647.71 \pm 730.91$ & 2584.94 (932.09) & $3096.67 \pm 1685.29$ & $2924.19(1153.71)$ & 0.002 \\
\hline Cotinine, $\mathrm{ng} \mathrm{ml}^{-1}$ & $20.46 \pm 51.15$ & $0.00(5.00)$ & $23.86 \pm 80.36$ & $0.00(0.00)$ & 0.651 \\
\hline$\gamma \mathrm{GT}, \mathrm{UI}^{-1}$ & $62.54 \pm 75.03$ & $40.11(40.82)$ & $24.36 \pm 23.73$ & $17.00(16.00)$ & $<0.001$ \\
\hline C-reactive protein, $\mathrm{mgI}^{-1}$ & $8.49 \pm 10.13$ & $4.95(7.84)$ & $2.89 \pm 3.33$ & $1.50(2.61)$ & $<0.001$ \\
\hline $\mathrm{IL}-6, \mathrm{pg} \mathrm{ml^{-1 }}$ & $2.18 \pm 8.23$ & $1.11(0.91)$ & $1.11 \pm 0.88$ & $0.85(0.83)$ & 0.08 \\
\hline TNF- $\alpha, \mathrm{pg} \mathrm{ml}^{-1}$ & $2.99 \pm 3.25$ & $2.56(2.76)$ & $1.82 \pm 1.94$ & $1.30(2.70)$ & $<0.001$ \\
\hline Temperature tympanum, ${ }^{\circ} \mathrm{C}$ & $36.62 \pm 0.41$ & $36.60(0.50)$ & $36.47 \pm 0.33$ & $36.40(0.50)$ & $<0.001$ \\
\hline \multicolumn{6}{|l|}{ Cardiac troponins } \\
\hline 24-h PP, mm Hg & $49 \pm 9$ & $48(10)$ & $47 \pm 7$ & $47(10)$ & 0.03 \\
\hline 24-h ST counts ( $N \%$ ) & $6 \pm 15(83 ; 53.21)$ & $1(5)$ & $3 \pm 6(77 ; 42.08)$ & $0(2)$ & 0.006 \\
\hline NT-proBNP, pg ml-1 & $44.78 \pm 48.96$ & $27.70(36.30)$ & $43.29 \pm 45.41$ & $32.35(30.17)$ & 0.775 \\
\hline ECG-LVH > $244.0 \mathrm{mV} . \mathrm{ms}$ & $69.52 \pm 41.38$ & $61.32(48.44)$ & $49.66 \pm 28.49$ & $44.18(41.02)$ & $<0.001$ \\
\hline Hypertensive, $N(\%)$ & $84(53.85)$ & - & $45(24.59)$ & - & $<0.001$ \\
\hline \multicolumn{6}{|l|}{ Medications } \\
\hline Hypercholesterolemia, N (\%) & $2(1.28)$ & - & $6(3.28)$ & - & 0.227 \\
\hline Hypertension, $N(\%)$ & $52(33.33)$ & - & $16(8.74)$ & - & $<0.001$ \\
\hline
\end{tabular}

Abbreviations: DBP, diastolic blood pressure; ECG-LVH, cornell product left ventricular hypertrophy; $\gamma$ GT, gamma-glutamyl transferase; IL, interleukin; NT-proBNP, N-terminal pro-Brain natriuretic peptide; SBP, systolic blood pressure; ST events, number of ischemic events in 24-h; TNF- $\alpha$, tumor necrosis factor-alpha; Trop T, troponin T.

$P$ values refer to independent $t$-tests.

Data are presented as mean \pm s.d. or number of participants $(\%)$.

stress. A resting 12-lead ECG was recorded with a Norav NHH-1200 ECG (NORAV Medical LTD PC 1200, Yokneam, Israel, Software version 5.030) and the ECG left ventricular hypertrophy (Cornell product, [RaVL+SV3]. $\times$ QRS duration) was determined. Values exceeding $244 \mathrm{mV} . \mathrm{ms}$ were indicative of $\mathrm{LVH}^{30}$

Statistical analyses. Statistical analyses were performed with Statistica version 12 (Statsoft Inc., Tulsa, OK, USA). Variables with skewed distributions ( $\gamma$-GT, cotinine and CRP) were log-transformed. Independent $t$-tests were used to compare the characteristics of the two ethnic groups. Chi-square tests $\left(\mathrm{X}_{2}\right)$ were used to determine prevalence as well as proportions. The a priori covariates used in all the statistical analyses were age, physical activity, body surface area, cotinine and $\gamma$-GT.

Single two-way general linear model interactions of the main effects (ethnicity $\times$ gender) were computed to determine differences in 24-h BP, cardiac remodeling, inflammation, cardiac troponin, silent ischemia and independent of a priori covariates in the bi-ethnic cohort. Subsequently, one-way analysis of covariance was performed to compare the differences in ethnic gender groups, adjusting for a priori covariates.

Multivariate linear regression analyses were performed. Variance inflation factors (all <2.5) indicated that there was no concern for multicollinearity between inflammatory markers when using adjusted $\mathrm{R}^{2}$ modeling as a prediction model. We therefore included the three inflammatory markers in the same model. Forced entry stepwise forward regression analyses determined the associations among the dependent variables of 24-h BP (SBP and DBP), cardiac stress (NT-proBNP) and cardiac remodeling (ECG-LVH). Independent variables included inflammation (TNF $\alpha$, IL-6, log-CRP), cardiac troponin, silent ischemia and a priori selected covariates. Pulse pressure was added as an independent variable in cardiac remodeling analyses to represent hyperpulsatile pressure. ${ }^{15,16}$ For all of the above-mentioned analyses, significant values were noted when $\mathrm{R}^{2} \geqslant 0.25$ and $P \leqslant 0.05$. The $\mathrm{F}$ to enter was set at 2.5 .

\section{RESULTS}

The baseline characteristics and overall health profiles of all African and Caucasian participants are shown in Table 1. A lower body surface area $(P=0.014)$, lower physical activity $(P=0.002)$ and higher $\gamma$-GT levels $(P=0.001)$ were observed in Africans. The Africans also showed higher levels $(P<0.001)$ of inflammatory markers (CRP and TNF- $\alpha$ ), tympanum temperature, 24-h BP, Trop T and LVH values than their Caucasian counterparts. Compared with Caucasians, more Africans were hypertensive $(53.85$ vs. $24.59 \% ; P<0.001)$ and experienced a greater number of ischemic events $(6 \pm 15(1 ; 5) v s .3 \pm 6(0 ; 3))$.

A significant interaction between main effects (ethnicity $\times$ gender) was evident for $\mathrm{CRP}(\mathrm{F}(1,326), 5.43 ; P=0.02)$, 24-h $\mathrm{SBP}(\mathrm{F}(1,326)$, 7.85; $P=0.005), 24-\mathrm{h} \mathrm{DBP}(\mathrm{F}(1,326), 14.74 ; P<0.001)$ and $24-\mathrm{h} \mathrm{ST}$ events $(\mathrm{F}(1,326), 10.44 ; P=0.001)$; these results supported the stratification into specific ethnic-gender groups for further analysis.

Figure 2 shows that African men had higher CRP $(P<0.001)$, IL-6 $(P=0.01), 24 \mathrm{~h} \mathrm{BP}(P<0.001)$ and LVH $(P=0.02)$ levels, as well as a greater number of ischemic events $(P=0.003)$ than their Caucasian counterparts. As shown in Figure 3, there was a similar trend for African women with significantly higher CRP $(P<0.001)$, TNF- $\alpha$ $(P=0.02), 24 \mathrm{~h}$ BP $(P<0.001), 24 \mathrm{~h}$ PP $(P<0.05)$ and $\mathrm{LVH}$ $(P<0.001)$ levels than their Caucasian counterparts. However, no differences were observed in the occurrence of ST events in the two ethnic female groups. 


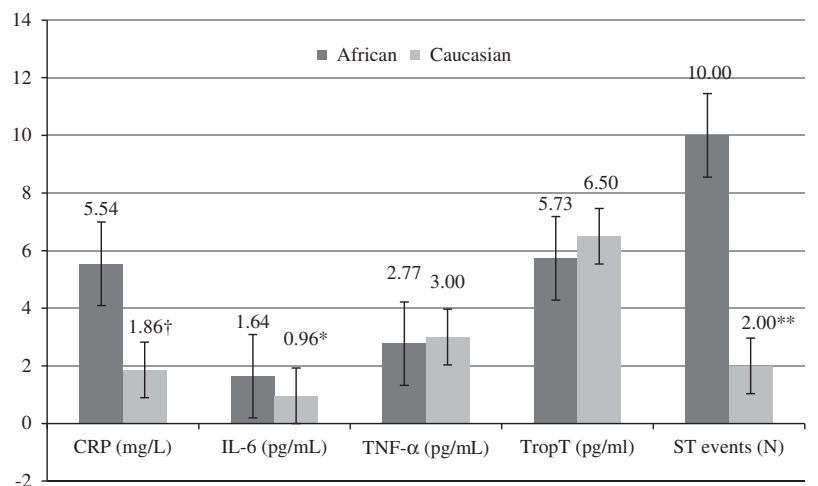

b

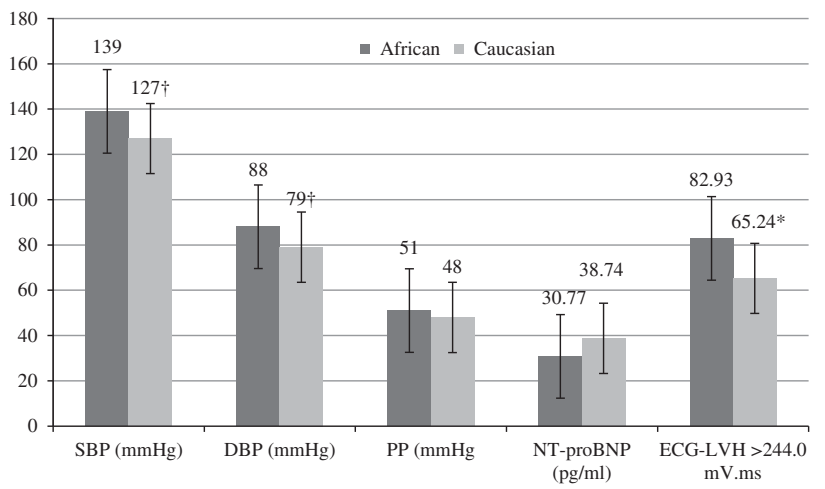

Figure 2 Results are reported (mean (s.d.)) for comparing adjusted differences in African vs. Caucasian men for inflammation measures, cardiac troponin and number of ischemic events (a), blood pressure and cardiac remodeling (b) values. Variables are adjusted age, body surface area, physical activity, log gamma glutamyl transferase $(\gamma-\mathrm{GT})$ and cotinine. ${ }^{*} P \leqslant 0.05 ;{ }^{* *} P \leqslant 0.01 ;{ }^{\dagger} P \leqslant 0.001$.

No significant associations were found among BP, cardiac remodeling and independent covariates in African and Caucasian women. Therefore, only associations in men will be discussed, as represented in Table 2. Overall, 24-h BP was associated $(P<0.05)$ with Trop T in all men. In Caucasian men, DBP was also associated with CRP $(\beta=0.25$; $95 \%$ confidence interval (CI) 0.06 to $0.45 ; P=0.013$ ). In African men, there were positive associations of cardiac stress (NT-proBNP) with TNF- $\alpha \quad(\beta=0.32 ; 95 \%$ CI 0.14 to $0.49 ; P<0.001))$ and Trop T $(\beta=0.40 ; 95 \%$ CI 0.18 to $0.63 ; P<0.001)$, as well as 24 -h PP $(\beta=0.22$; $95 \%$ CI 0.06 to $0.33 ; P=0.048)$. LVH was also associated with $24-\mathrm{h}$ hyperpulsatile pressure in the African male cohort only ( $\beta=0.44 ; 95 \%$ CI 0.18 to $0.69 ; P=0.001)$.

\section{DISCUSSION}

The main aim of our study was to determine whether inflammation and myocardial injury are associated with cardiac remodeling in a bi-ethnic male and female cohort from South Africa. We found significant associations of cardiac stress (NT-proBNP) with inflammation (TNF- $\alpha$ ) and Trop T, as well as PP, in African men.

As a marker of cardiac stress, NT-proBNP is a natriuretic peptide produced by the cardiac myocytes and fibroblasts in response to cardiac wall stress. ${ }^{18,19,31}$ Inflammation can alter vascular function and lead to an increase in cardiac wall stress through multiple pathways related to myocardial remodeling. $1,13,18,32$ The inflammatory marker TNF- $\alpha$ contributes to apoptosis, and it may also lead to endothelial dysfunction by enhancing NO inactivity. ${ }^{33-36}$ Indeed, a greater degree a

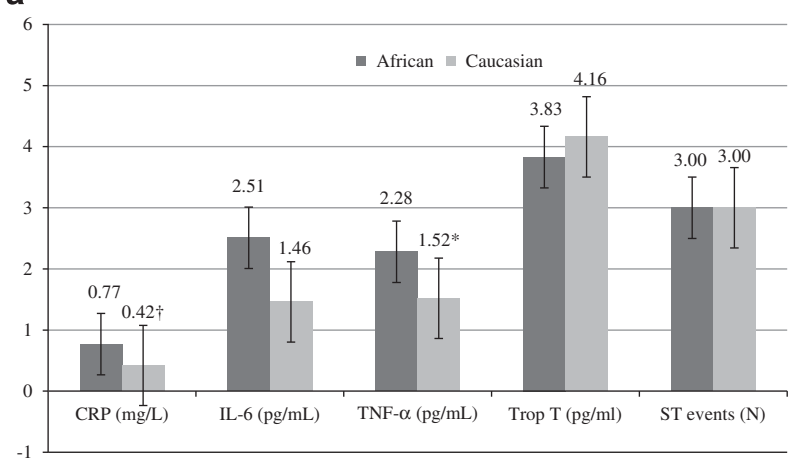

b

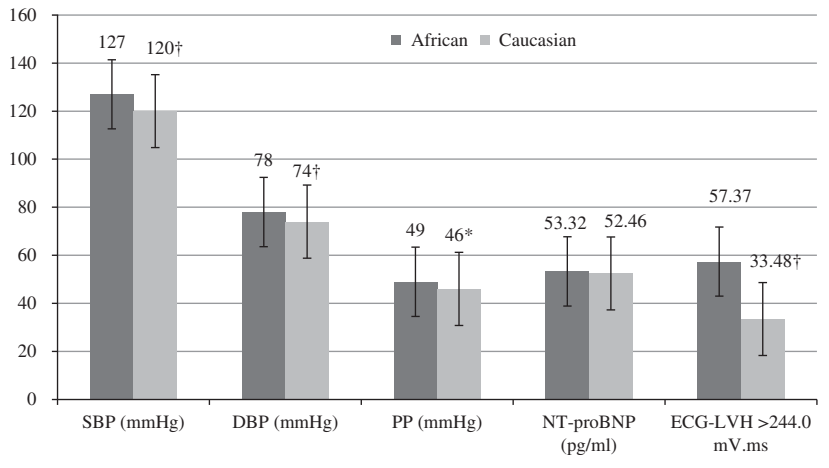

Figure 3 Results are reported (mean (s.d.)) for comparing adjusted differences in African vs. Caucasian women for inflammation measures, cardiac troponin and number of ischemic events (a), blood pressure and cardiac remodeling (b) values. Adjustment was made for age, body surface area, physical activity, log gamma glutamyl transferase $(\gamma-\mathrm{GT})$ and cotinine. ${ }^{*} P \leqslant 0.05 ; * * P \leqslant 0.01 ;{ }^{\dagger} P \leqslant 0.001$.

of endothelial dysfunction was observed in both African-Americans and Africans. ${ }^{4,37,38}$ Attenuated vasodilation that contributes to excessive vasoconstriction and $\alpha$-adrenergic vascular responsiveness in Africans may contribute to an increased afterload on the myocardium, a potent stimulus for cardiac remodeling. ${ }^{8,12}$ Ambulatory SBP, as well as PP, have previously been shown to be associated with cardiac wall remodeling in 75 African men with low-grade inflammatory status (>3 mg $1^{-1}$ hs-CRP). ${ }^{1}$ In our study, hyperdynamic BP and inflammation both acted as factors that might contribute to structural wall abnormalities. The differences in inflammation and BP between Africans and Caucasians in our cohort may support the notion of differing mechanisms contributing to remodeling in various ethnic groups.

The changes that may contribute to an increase in cardiac wall stress include not only myocyte hypertrophy but also myocyte death and modifications of the ECM, thus explaining the association between NT-proBNP and Trop T in African men. ${ }^{8,39,40}$ Myocyte necrosis is a passive unregulated process of cell death that occurs as a result of cardiac injury and leads to the release of Trop $\mathrm{T}$ owing to the loss of cell membrane integrity and the degradation of the contractile apparatus. ${ }^{8-10}$ Elevations in Trop $\mathrm{T}$ have previously been associated with African ethnicity in Americans. ${ }^{11}$ Although our study revealed no differences in Trop T levels between African and Caucasian individuals, we showed that Trop $\mathrm{T}$ was associated with BP in African and Caucasian men. The association between BP and ischemic events in African men can thus explain how a decrease in metabolic supply to the myocardial tissue due to hypertension results in hypoxia of the myocardium. ${ }^{14}$ Persistent ischemia may result in irreversible cell death 
Table 2 Independent associations between blood pressure, cardiac troponins, ECG left ventricular hypertrophy and coronary artery disease risk markers in African and Caucasian men

\begin{tabular}{|c|c|c|c|c|c|c|c|c|}
\hline & $\begin{array}{c}S B P \\
\beta(95 \% \mathrm{Cl})\end{array}$ & $\begin{array}{c}D B P \\
\beta(95 \% \mathrm{Cl})\end{array}$ & $\begin{array}{l}N T \text {-proBNP } \\
\beta(95 \% \mathrm{Cl})\end{array}$ & $\begin{array}{c}L V H \\
\beta(95 \% \mathrm{Cl})\end{array}$ & $\begin{array}{c}S B P \\
\beta(95 \% \mathrm{Cl})\end{array}$ & $\begin{array}{c}D B P \\
\beta(95 \% \mathrm{Cl})\end{array}$ & $\begin{array}{l}N T-p r o B N P \\
\beta(95 \% \mathrm{Cl})\end{array}$ & $\begin{array}{c}L V H \\
\beta(95 \% \mathrm{Cl})\end{array}$ \\
\hline Adjusted $\mathrm{R}^{2}$ & 0.36 & 0.24 & 0.45 & 0.30 & 0.23 & 0.29 & 0.08 & 0.07 \\
\hline TNF- $\alpha$ & NS & NS & $0.32^{\dagger}(0.14 ; 0.49)$ & NS & NS & NS & NS & NS \\
\hline IL-6 & NS & NS & NS & NS & NS & NS & NS & NS \\
\hline Trop T & $0.29 *(0.26 ; 0.52)$ & $0.26 *(0.05 ; 0.47)$ & $0.40^{\dagger}(0.18 ; 0.63)$ & NS & $0.23 *(0.04 ; 0.41)$ & $0.19 *(0.01 ; 0.37)$ & NS & NS \\
\hline ST events & NS & NS & NS & NS & NS & NS & NS & NS \\
\hline Pulse pressure & - & - & $0.22 *(0.06 ; 0.33)$ & $0.44^{* *}(0.18 ; 0.69)$ & - & - & NS & NS \\
\hline
\end{tabular}

Abbreviations: CI, confidence interval; DBP, diastolic blood pressure; IL-6, Interleukin-6; log-CRP, C-reactive protein; LVH, left ventricular hypertrophy; NT-proBNP, N-terminal pro-Brain Natriuretic peptide; ST events, number of ischemic events in $24 \mathrm{~h}$; SBP, systolic blood pressure; TNF- $\alpha$, tumor necrosis factor-alpha; Trop T, Troponin T.

Independent variables included age, body surface area, physical activity, $\log \gamma$-GT and log cotinine.

${ }^{*} P \leqslant 0.05 ;{ }^{* *} P \leqslant 0.01 ;{ }^{\dagger} P \leqslant 0.001$

and thus lead to the release of Trop T. Indeed, Malan et al. ${ }^{13}$ have shown that silent ischemia and $\mathrm{LVH}$ are facilitated by vascular responsiveness in African men. The sympatho-excitatory effects of indirect markers of the sympathetic nervous system activity, such as increased heart rate, 24-h silent ischemic events and chronic hyperglycemia, which has been shown in other SABPA sub-studies in the African cohort, may thus potentiate adrenergic overdrive and reinforce metabolic overdrive and structural alterations. ${ }^{41-43}$ The contribution of inflammation to the pathogenesis of endothelial dysfunction can also result in reduced blood flow that further contributes to the development of ischemia-related cardiac cell death and thus cardiac remodeling. ${ }^{3,33,44}$

The increased pro-inflammatory profile has previously been reported in our African cohort. ${ }^{1,45}$ However, the mean values of the inflammatory cytokines in the Africans (IL-6: 2.18 and TNF- $\alpha$ : $2.99 \mathrm{pg} \mathrm{ml}^{-1}$ ) were higher than the reference ranges reported by Todd et al. (IL-6: 1.89 and TNF- $\alpha, 1.51 \mathrm{pg} \mathrm{ml}^{-1}$ ). ${ }^{46}$ Because these values were accompanied by a higher mean PP, the risk of vascular wall permeability may be increased, because studies have shown that inflammation can lead to an increase in arterial stiffness. ${ }^{15,16,47}$ The resulting increases in ventricular transmural pressure and volume overload are major determinants of cardiac stretching, and they induce cardiac remodeling. ${ }^{48}$ The pro-inflammatory cytokine IL- 6 influences the coagulation cascade by increasing tissue factor and von Willebrand factor production, which may further lead to an increase in collagen formation. ${ }^{49}$ Indeed, higher resting levels of the von Willebrand factor have been reported in Africans compared with Caucasians, thus supporting the mechanisms by which thrombus formation might be promoted through the actions of IL- 6 on hemostasis. ${ }^{34,49,50}$ TNF- $\alpha$ seems to induce the production of reactive oxygen species, thus resulting in oxidative stress that may mediate the production of various adhesion molecules. ${ }^{2,51}$ Vascular responsiveness and depressed heart rate variability in Africans support the possibility of increased oxidative stress in our cohort. ${ }^{13,41}$ Indeed, lower levels of glutathione concentrations are indicative of oxidative stress and are associated with subclinical atherosclerosis in Africans. ${ }^{52}$ This process contributes to the structural changes in the arterial wall that may contribute to arterial stiffness and an increased workload of the myocardium.

Multiple studies have shown that NT-proBNP levels are higher in African men than in Caucasian men; ${ }^{20-22}$ however, these studies did not consider socioeconomic status. In our study of African and Caucasian men with similar socioeconomic status, we were unable to replicate this finding. Our findings may suggest chronic activation of the sympathetic nervous system in individuals exposed to overdemanding situations, which may be one explanation for the previously observed ethnic differences in NT-proBNP levels. Although $\mathrm{BP}$ and LVH were increased in African women compared with Caucasian women, we did not find significant associations of cardiac troponin and inflammatory markers with cardiac remodeling in the female cohort. A pro-inflammatory profile was observed in both men and women of African ethnicity. One possibility for the absence of associations in the female subgroup could be the higher BP observed in the African men than in the women. The men also consumed significantly higher amounts of alcohol than their female counterparts, as evidenced by the elevated $\gamma \mathrm{GT}$ values, which may alter vascular tone and affect subclinical vascular remodeling. ${ }^{41,45}$

Our study has several limitations. The main limitation is the cross-sectional design of the study because only follow-up data could provide insight into the progression of end organ damage and the influence of inflammation. This study represents a cohort of only the South African population; therefore, the findings may not be applicable to the entire South African population. The observed significant associations nevertheless provide valuable information in terms of the increased risk of cardiovascular disease of African men.

In conclusion, our hypothesis was only partially supported, in that African men, but not African women, showed associations among hyperpulsatile pressure, inflammation, cardiac stress and remodeling (LVH). We demonstrated an association of inflammation and cardiac troponin with cardiac remodeling in African men. Related risk markers of chronic low-grade systemic inflammation, arterial tone and cardiac troponin may further underscore the possibility of impeded myocardial oxygen supply in African men. The susceptibility for cardiac wall remodeling increases with hyperpulsatile pressure, low-grade systemic inflammation and ventricular stress, which may lead to the development of future cardiovascular events in these men.

\section{CONFLICT OF INTEREST}

The authors declare no conflict of interest.

\section{ACKNOWLEDGEMENTS}

This study would not have been possible without all the participants who volunteered to be part of the SABPA study. We would like to thank the following institutions that provided financial support: North-West University, Potchefstroom, South Africa; the National Research Foundation (NRF); the Department of Education, North-West Province, South Africa; ROCHE diagnostics; and the Metabolic Syndrome Institute, France. Any opinion, 
findings and conclusions or recommendations expressed in this material are those of the author(s); therefore, funders do not accept any liability with regard to this study.

1 Van der Walt C, Malan L, Uys AS, Malan NT. Low grade inflammation and ECG left ventricular hypertrophy in urban African males: the SABPA study. Heart Lung Circ 2013; 22: 924-929.

2 Volpato S, Guralnik JM, Ferrucci L, Balfour J, Chaves P, Fried LP, Harris TB. Cardiovascular disease, interleukin-6, and risk of mortality in older women the women's health and aging study. Circulation 2001; 103: 947-953.

3 Kofler S, Nickel T, Weis M. Role of cytokines in cardiovascular diseases: a focus on endothelial responses to inflammation. Clin Sci (Lond) 2005; 108: 205-213.

4 Brown MD, Feairheller DL, Thakkar S, Veerabhadrappa P, Park J-Y. Racial differences in tumour necrosis factor- $\alpha$-induced endothelial microparticles and interleukin-6 production. Vasc Health Risk Manag 2011; 7: 541.

5 Pearson TA, Mensah GA, Alexander RW, Anderson JL, Cannon RO, Criqui M, FadI YY, Fortmann SP, Hong Y, Myers GL, Rifai N, Smith SC, Taubert K, Tracy RP, Vinicor F. Markers of inflammation and cardiovascular disease application to clinical and public health practice: a statement for healthcare professionals from the centers for disease control and prevention and the American Heart Association. Circulation 2003; 107: 499-511.

6 Figiel I. Pro-inflammatory cytokine TNF-alpha as a neuroprotective agent in the brain Acta Neurobiol Exp (Wars) 2008; 68: 526-534.

7 Krown KA, Page MT, Nguyen C, Zechner D, Gutierrez V, Comstock KL, Glembotski CC, Quintana PJ, Sabbadini RA. Tumour necrosis factor alpha-induced apoptosis in cardiac myocytes. Involvement of the sphingolipid signaling cascade in cardiac cell death J Clin Invest 1996; 98: 2854.

8 Del Carlo CH, O'Connor CM. Cardiac troponins in congestive heart failure. Am Heart $J$ 1999; 138: 646-653.

9 Korff S, Katus HA, Giannitsis E. Differential diagnosis of elevated troponins. Heart 2006; 92: 987-993.

10 Muthu V, Kozman H, Liu K, Smulyan H, Villarreal D. Cardiac troponins: bench to bedside interpretation in cardiac disease. Am J Med Sci 2014; 347: 331-337.

11 Wallace TW, Abdullah SM, Drazner MH, Das SR, Khera A, McGuire DK, Wians F, Sabatine MS, Morrow DA, de Lemos JA. Prevalence and determinants of troponin T elevation in the general population. Circulation 2006; 113: 1958-1965.

12 Sibiya MJ, Norton GR, Hodson B, Redelinghuys M, Maseko MJ, Majane OH, Libhaber E, Woodiwiss AJ. Gender-specific contribution of aortic augmentation index to variations in left ventricular mass index in a community sample of African ancestry. Hypertens Res 2014; 37: 1021-1027.

13 Malan L, Hamer M, Schlaich MP, Lambert GW, Harvey BH, Reimann M, Ziemssene T, de Geus EJ, Huisman HW, van Rooyen JM, Schutte R, Schutte AE, Fourie CMT, Seedat YK, Malan NT. Facilitated defensive coping, silent ischaemia and ECG left-ventricular hypertrophy: the SABPA study. J Hypertens 2012; 30: 543-550.

14 Mill J, Stefanon I, Dos Santos L, Baldo M. Remodelling in the ischemic heart: the stepwise progression for heart failure. Braz J Med Biol Res 2011; 44: 890-898.

15 Van Bussel BC, Schouten F, Henry RM, Schalkwijk CG, de Boer MR, Ferreira I, Smulders YM, Twisk JW, Stehouwer CD. Endothelial dysfunction and low-grade inflammation are associated with greater arterial stiffness over a 6 -year period. Hypertension 2011; 58: 588-595.

16 Dart AM, Kingwell BA. Pulse pressure - a review of mechanisms and clinical relevance. J Am Coll Cardiol 2001; 37: 975-984.

17 Lorell BH, Carabello BA. Left ventricular hypertrophy pathogenesis, detection, and prognosis. Circulation 2000; 102: 470-479.

18 Ismaiel JY, Moustafa SR. Association of $\mathrm{N}$-terminal pro-brain natriuretic peptide, matrix -metalloprotinase-9, and 8-0xo-2 deoxy guanosine with the incidence of essential hypertension. Am J Int Med 2015; 3: 67-77.

19 Hall C. NT-ProBNP: the mechanism behind the marker. J Card Fail 2005; 11: S81-S83.

20 Kruger R, Schutte R, Huisman H, Argraves W, Rasmussen LM, Olsen M, Schutte AE. NT-proBNP is associated with fibulin-1 in Africans: The SAfrEIC study. Atherosclerosis 2012; 222: 216-221.

21 Kruger R, Schutte R, Huisman HW, Hindersson P, Olsen MH, Schutte AE. N-terminal prohormone B-type natriuretic peptide and cardiovascular function in Africans and Caucasians: the SAfrEIC study. Heart Lung Circ 2012; 21: 88-95.

22 Malan L, Hamer M, Frasure-Smith N, Steyn HS, Malan NT. COHORT PROFILE: Sympathetic activity and Ambulatory Blood Pressure in Africans (SABPA) prospective cohort study. Int J Epidemiol 2015; 44: 1814-1822.

23 Mancia G, Fagard R, Narkiewicz K, Redon J, Zanchetti A, Böhm M, Christiaens T, Cifkova R, De Backer G, Dominiczak A, Galderisi M, Grobbee DE, Jaarsma T, Kirchhof P, Kjeldsen SE, Laurent S, Manolis AJ, Nilsson PM, Ruilope LM, Schmieder RE, Sirnes PA, Sleight P, Viigimaa M, Waeber B, Zannad F. 2013 $\mathrm{ESH} / \mathrm{ESC}$ guidelines for the management of arterial hypertension: the Task Force for the Management of Arterial Hypertension of the European Society of Hypertension (ESH) and of the European Society of Cardiology (ESC). Blood Press 2013; 22 . 193-278.

24 World Medical Association. World Medical Association Declaration of Helsinki: ethical principles for medical research involving human subjects. JAMA 2013; 310 2191
25 Mosteller R. Simplified calculation of body-surface area. N Engl J Med 1987; 317: 1098.

26 Benowitz NL, Bernert JT, Caraballo RS, Holiday DB, Wang J. Optimal serum cotinine levels for distinguishing cigarette smokers and nonsmokers within different racial/ethnic groups in the United States between 1999 and 2004. Am J Epidemiol 2009; 169: 236-248.

27 Tsai J, Ford ES, Li C, Zhao G. Past and current alcohol consumption patterns and elevations in serum hepatic enzymes among US adults. Addict Behav 2012; 37: 78-84.

28 Croghan C, Egeghy P. Methods of dealing with values below the limit of detection using SAS. Southern SAS User Group 2003: 22-24.

29 Uen S, Fimmers R, Weisser B, Balta O, Nickenig G, Mengden T. ST-segment depression in hypertensive patients: A comparison of exercise test versus Holter ECG. Vasc Health Risk Manag 2008; 4: 107301080.

30 Ishikawa J, Ishikawa S, Kabutoya T, Gotoh T, Kayaba K, Schwartz JE, Pickering TG, Shimada K, Kario K. Cornell product left ventricular hypertrophy in electrocardiogram and the risk of stroke in a general population. Hypertension 2009; 53: 28-34.

31 Hall C. Essential biochemistry and physiology of (NT-pro) BNP. Eur J Heart Fail 2004; 6: 257-260.

32 Takeshita Y, Watanabe S, Hattori T, Nagasawa K, Matsuura N, Takahashi K, Murohara T, Nagata K. Blockade of glucocorticoid receptors with RU486 attenuates cardiac damage and adipose tissue inflammation in a rat model of metabolic syndrome. Hypertens Res 2015; 38: 741-750.

33 Blake GJ, Ridker PM. Novel clinical markers of vascular wall inflammation. Circ Res 2001; 89: 763-771.

34 Pauletto P, Rattazzi M. Inflammation and hypertension: the search for a link. Nephrol Dial Transplant 2006; 21: 850-853.

35 Boos CJ, Lip GY. Is hypertension an inflammatory process? Curr Pharma Des 2006; 12 $1623-1635$.

36 Koenig W, Sund M, Fröhlich M, Fischer H-G, Löwel H, Döring A, Hutchinson WL, Pepys MB. C-reactive protein, a sensitive marker of inflammation, predicts future risk of coronary heart disease in initially healthy middle-aged men results from the MONICA (Monitoring Trends and Determinants in Cardiovascular Disease) Augsburg Cohort Study, 1984 to 1992. Circulation 1999; 99: 237-242.

37 Kalinowski L, Dobrucki IT, Malinski T. Race-specific differences in endothelial function predisposition of African Americans to vascular diseases. Circulation 2004; 109 2511-2517.

38 Reimann M, Hamer M, Malan NT, Schlaich MP, Lambert GW, Ziemssen T, Böger R, Malan L. Effects of acute and chronic stress on the L-arginine nitric oxide pathway in black and white South Africans: the SABPA study. Psychosom Med 2013; 75: 751-758.

39 Shih $\mathrm{H}$, Lee B, Lee RJ, Boyle AJ. The aging heart and post-infarction left ventricular remodelling. J Am Coll Cardiol 2011; 57: 9-17.

40 Cohn JN, Ferrari R, Sharpe N. Cardiac remodelling - concepts and clinical implications: a consensus paper from an international forum on cardiac remodelling. J Am Coll Cardiol 2000; 35: 569-582.

41 Malan L, Hamer M, Schlaich M, Lambert G, Ziemssen T, Reimann M, Frasure-Smith N, Amirkhan JH, Schutte R, van Rooyen JM, Mels CM, Fourie CM, Uys AS, Malan NT. Defensive coping facilitates higher blood pressure and early sub-clinical structural vascular disease via alterations in heart rate variability: the SABPA study. Atherosclerosis 2013; 227: 391-397.

42 Hamer M, Malan L. Psychophysiological risk markers of cardiovascular disease. In: Psychophysiological Biomarkers of Health. Neurosci Biobehav Rev 2010; 35: 76-83.

43 Malan L, Hamer M, Schlaich MP, Lambert GW, Ziemssen T, Reimann M, Steyn HS, Schutte R, Smith W, van Rooyen JM, Fourie CMT, Malan NT. Defensive active coping facilitates chronic hyperglycemia and endothelial dysfunction in African men: the SABPA study. Int J Cardiol 2013; 168: 999-1005.

44 Libby P, Ridker PM, Maseri A. Inflammation and atherosclerosis. Circulation 2002; 105: 1135-1143.

45 Hamer M, Malan L, Schutte A, Huisman H, van Rooyen J, Schutte R, Fourie CMT, Malan NT, Seedat YK. Conventional and behavioral risk factors explain differences in sub-clinical vascular disease between black and Caucasian South Africans: The SABPA study. Atherosclerosis 2011; 215: 237-242.

46 Todd J, Simpson P, Estis J, Torres V, Wub AH. Reference range and short- and longterm biological variation of interleukin (IL)-6, IL-17A and tissue necrosis factor-alpha using high sensitivity assays. Cytokine 2013; 64: 660-665.

47 Hayman DM, Xiao Y, Yao Q, Jiang Z, Lindsey ML, Han H-C. Alterations in pulse pressure affect artery function. Cell Mol Bioeng 2012; 5: 474-487.

48 Shyu K. Cellular and molecular effects of mechanical stretch on vascular cells and cardiac myocytes. Clin Sci 2009; 116: 377-389.

49 Wannamethee S, Whincup P, Rumley A, Lowe G. Inter-relationships of interleukin-6, cardiovascular risk factors and the metabolic syndrome among older men. J Thromb Haemost 2007; 5: 1637-1643.

50 Von Känel R, Hamer M, Malan NT, Scheepers JD, Meiring M, Malan L. Procoagulant reactivity to laboratory acute mental stress in Africans and Caucasians, and its relation to depressive symptoms: The SABPA Study. Thromb Haem 2013; 110: 977-986.

51 Savoia C, Schiffrin EL. Inflammation in hypertension. Curr Opin Nephrol Hypertens 2006; 15: 152-158.

52 Schutte R, Schutte AE, Huisman HW, van Rooyen JM, Malan NT, Péter S, Fourie CMT, van der Westhuizen, Louw R, Botha CA, Malan L. Blood glutathione and sub-clinical atherosclerosis in African men: the SABPA study. Am J Hypertens 2009; 22 : 1154-1159. 